\title{
Fibrosing mediastinitis as a rare mechanism of pulmonary oedema in sarcoidosis
}

\section{To the Editors:}

Pulmonary oedema in sarcoidosis can usually be attributed to congestive heart failure or veno-occlusive disease. Here we report a rare case of pulmonary oedema, which was due to pulmonary vein stenosis secondary to fibrosing mediastinitis.

Our patient was a 44-yr-old female who was admitted to our hospital because of acute dyspnoea. She had a histogically confirmed cutaneous sarcoidosis diagnosed $17 \mathrm{yrs}$ prior to presentation at our hospital and had received aminoquinolins for 9 yrs. 1-yr prior to presentation at our institute, pulmonary involvement was diagnosed based on clinical complaints (progressive dyspnoea and non-productive cough) and diffuse pulmonary infiltration with bilateral hilar enlargement on chest radiography. On admission to our hospital, the patient was orthopnoeic and polypnoeic, and had dilatation of jugular veins suggesting congestive cardiac failure. Arterial blood gases in room air showed hypoxaemia (an arterial oxygen tension level of $62 \mathrm{mmHg}$ ) and hypocapnia (a carbon dioxide arterial tension of $30 \mathrm{mmHg}$ ). Transthoracic echocardiography showed right ventricular dilatation and high systolic pulmonary artery pressure at $45 \mathrm{mmHg}$, while the left ventricular function appeared normal. Pulmonary ventilation-perfusion scintigraphy showed mismatched perfusion defects in the upper left lobe and matched ventilation-perfusion defects in the right lung. Contrast-enhanced computed tomography (CT) (fig. 1) showed:

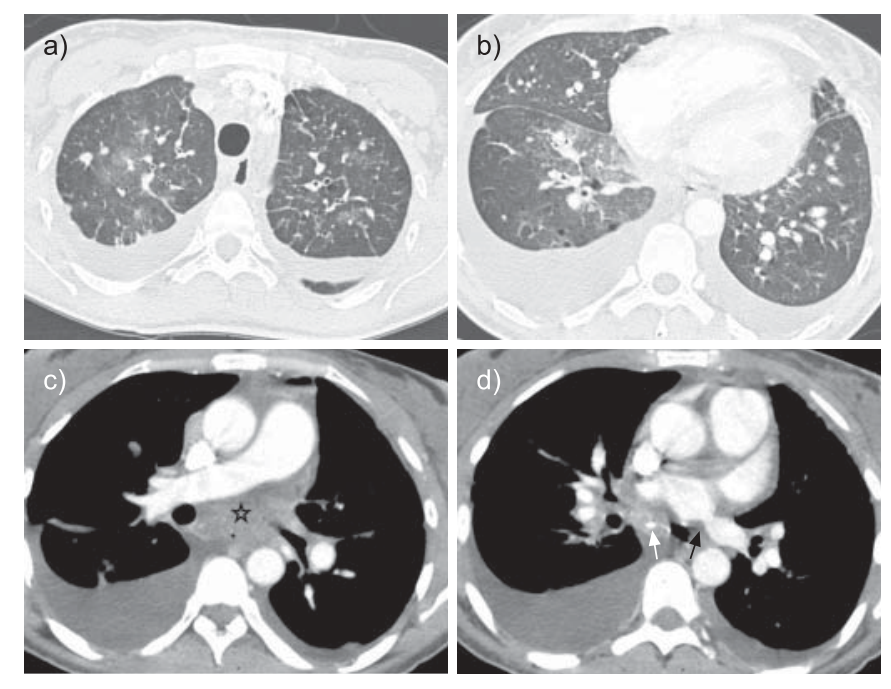

FIGURE 1. Contrast-enhanced computed tomography at admission showing signs of $a$ and b) parenchymal and pleural signs of hydrostatic pulmonary oedema (lung window settings image), including central ground-glass attenuation, bilateral pleural effusion and thickening of interlobular septa in the upper lobes. c and d) A soft-tissue attenuation mass (star) with foci of calcifications (white arrow) was noted (mediastinal window settings image) without contrast enhancement of the left superior (c) and right inferior pulmonary veins and stenoses of the left inferior pulmonary vein (black arrow) (d) soft-tissue attenuation mediastinal masses with scare foci of calcifications; varying degrees of stenosis of the left superior and the right inferior pulmonary veins, and near total occlusion of the left superior and the right inferior pulmonary vein at their mediastinal level, without any obstruction of pulmonary arteries; parenchymal and pleural signs, all typical of hydrostatic pulmonary oedema (ground-glass attenuation, thickening of interlobular septa and bilateral pleural effusion) (fig. 1a and b); and absence of thrombosis of pulmonary arteries. Pulmonary function showed a total lung capacity of $74 \%$ predicted, a vital capacity of $70 \%$ pred, forced expiratory volume in $1 \mathrm{~s}$ of $65 \%$ pred and a transfer factor of the lung for carbon monoxide of $57 \%$ pred. Sarcoidosis fibrosing mediastinitis complicated by pulmonary oedema and pleural effusion due to pulmonary venous obstruction was diagnosed. Diuretics, anticoagulant therapy and oxygen were initiated, followed by steroid therapy (prednisone $1 \mathrm{mg} \cdot \mathrm{kg}^{-1} \cdot \mathrm{d}^{-1}$ ). Under treatment, a dramatic and rapid improvement of dyspnoea and a normalisation of haematosis were observed. After 1 month of treatment, a new CT scan showed regression of pleural effusion and lung groundglass attenuation, while all other abnormalities, particularly in the mediastinum and concerning pulmonary veins, remained unchanged. The treatment was continued for $1 \mathrm{yr}$ while tapering steroid therapy. Transthoracic echocardiography showed pulmonary hypertension with a systolic pulmonary artery pressure of $40 \mathrm{mmHg}$ and a normalisation of right ventricular volume. $1 \mathrm{yr}$ later, pulmonary function remained unchanged, while a thoracic CT scan showed a slightly reduced soft-tissue attenuation mediastinal mass with an increased number of calcifications (fig. 2) and no sign of pulmonary oedema or pleural effusion. Conversely, there was no change in the pulmonary vein stenosis.

To our knowledge, we report herein the first case of pulmonary oedema secondary to fibrosing mediastinitis-induced pulmonary vein obstruction in sarcoidosis. The diagnosis was supported by CT imaging evidencing pulmonary oedema, fibrosing mediastinitis and pulmonary vein stenosis. Congestive heart failure could be withdrawn according to echocardiography, and pulmonary embolism was ruled out despite confusing scintigraphy ventilation-perfusion mismatch. Interestingly, this mechanism is to be added to veno-occlusive disease as a non-cardiac cause of pulmonary oedema in sarcoidosis.

Fibrosing mediastinitis is most often due to causes other than sarcoidosis [1]: infectious diseases (histoplasmosis, tuberculosis, aspergillosis, blastomycosis and cryptococcosis), autoimmune disease, Behçet's disease, radiation therapy, Hodgkin's disease and idiopathic. Regarding sarcoidosis, DEVARAJ et al. [2] reported two cases in a series of 12 patients, with CT images and histology of fibrosing mediastinitis (16.6\% of patients). Vascular obstruction due to fibrosing mediastinitis is frequent [3-13], particularly during histoplasmosis. Superior vena cava obstruction is the most commonly reported and is easily diagnosed. Pulmonary artery obstruction has also been 

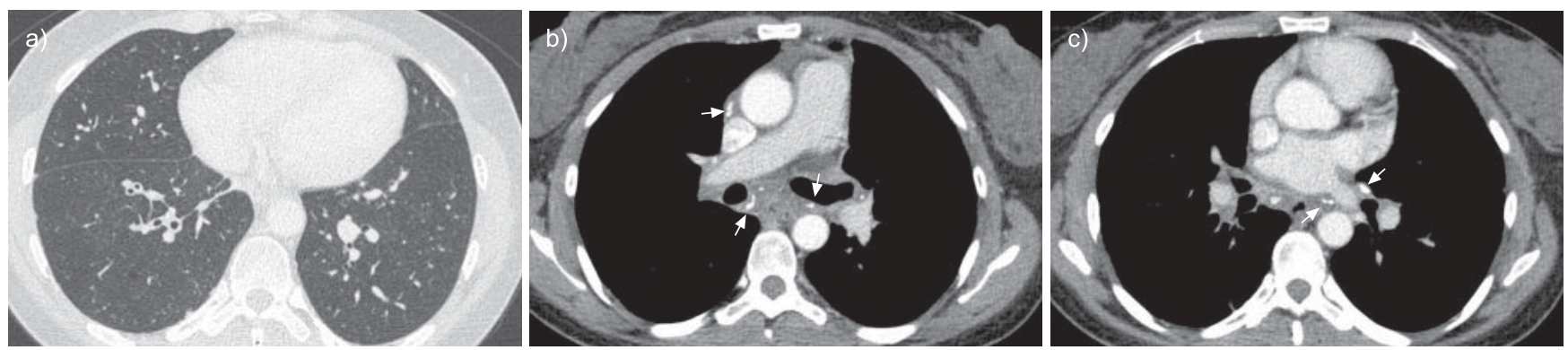

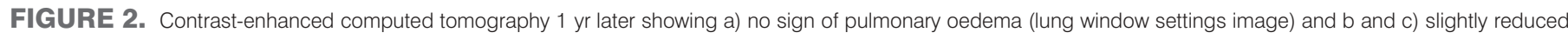

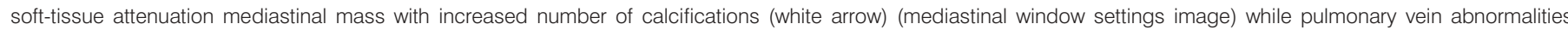
remained unchanged.

reported [3-6]. In sarcoidosis, two cases were reported by HAMILTON-CRAig et al. [7] who were successfully treated by stent placement in the pulmonary arteries. Conversely, there are only a few cases in the literature of pulmonary venous obstruction [10-13]. This happened in three of 64 patients $(4.6 \%)$ with fibrosing mediastinitis in the study by SCHOWENGERDT et al. [11]. Clinical manifestations include cough, progressive respiratory distress and haemoptysis. This pattern of symptoms has been called the "the pseudomitral stenosis syndrome" [1]. Pulmonary venous obstruction occurs gradually, so the symptoms may be delayed and the diagnosis is elusive. In the advanced stage of the disease with more than one pulmonary vein occluded, recurrent episodes of pulmonary oedema may occur and are frequently the cause of death [12-14].

There is no well-established treatment for fibrosing mediastinitis. With regard to the present case, we hypothesise that the rapid improvement after treatment was mainly attributable to diuretics. Conversely, steroids had little or no beneficial effect as pulmonary hypertension and pulmonary vein obstruction remained unchanged. Such absence of response to steroids has previously been reported in a case of pulmonary arterial stenosis secondary to sarcoidosis mediastinitis [15].

In conclusion, clinicians must be aware of this possible cause of acute respiratory insufficiency in sarcoidosis in which both thoracic CT and echocardiography appear crucial for establishing the diagnosis. In such a case, symptomatic treatment is mandatory but steroid therapy may be poorly efficient when fibrotic lesions in the mediastinum make up the main mechanism of pulmonary vein stenosis.

\section{F. Yangui ${ }^{* \#}$, J-P. Battesti ${ }^{*}$, D. Valeyre* ${ }^{*}$ A.B. Kheder ${ }^{\#}$ and P-Y. Brillet}

*Pulmonary Dept, and "Radiology Dept, University Paris 13, EA2363 and Assistance Publique-Hôpitaux de Paris, Hôpital Avicenne, Bobigny, France. " Dept of Pulmonology (Pavillon IV), Abderrahmane Mami Hospital, Ariana, Tunisia.

Correspondence: P-Y. Brillet, Service de Radiologie, Hôpital Avicenne, 125, route de Stalingrad, 93000 Bobigny Cedex, France. E-mail: pierre-yves.brillet@avc.aphp.fr
Statement of Interest: None declared.

\section{REFERENCES}

1 Rossi SE, McAdams HP, Rosado-de-Christenson ML, et al. Fibrosing mediastinitis. Radiographics 2001; 21: 737-757.

2 Devaraj A, Griffin N, Nicholson AG, et al. Computed tomography findings in fibrosing mediastinitis. Clin Radiol 2007; 62: 781-786.

3 Denlinger CE, Fernandez FG, Patterson GA, et al. Fibrosing mediastinitis associated with complete occlusion of the left main pulmonary artery. Ann Thorac Surg 2009; 87: 323.

4 Satpathy R, Aguila V, Mohiuddin SM, et al. Fibrosing mediastinitis presenting as pulmonary stenosis: Stenting works. Int J Cardiol 2007; 118: e85-e86.

5 Berry DF, Buccigrossi D, Peabody J, et al. Pulmonary vascular occlusion and fibrosing mediastinitis. Chest 1986; 89; 296-301.

6 Botticelli JT, Schlueter DP, Lange RL. Pulmonary venous and arterial hypertension due to chronic fibrous mediastinitis: hemodynamics and pulmonary function. Circulation 1966; 33; 862-871.

7 Hamilton-Craig CR, Slaughter R, McNeil K, et al. Improvement after angioplasty and stenting of pulmonary arteries due to sarcoid mediastinal fibrosis - case report. Heart Lung Circ 2009; 18: 222-225.

8 Doyle TP, Loyd JE, Robbins IM. Percutaneous pulmonary artery and vein stenting a novel treatment for mediastinal fibrosis. Am J Respir Crit Care Med 2001; 164: 657-660.

9 Thiessen R, Matzinger FR, Seely J, et al. Fibrosing mediastinitis: successful stenting of the pulmonary artery. Can Respir J 2008; 15: $41-44$.

10 Leong DP, Dundon BK, Steele PM. Unilateral pulmonary vein stenosis secondary to idiopathic fibrosing mediastinitis. Heart 2008; 94: 776.

11 Schowengerdt CC, Suyemoto R, Main FB. Granulomatous and fibrous mediastinitis. J Thorac Cardiovasc Surg 1969; 57: 365-379.

12 Routsi C, Charitos C, Rontogianni D, et al. Unilateral pulmonary edema due to pulmonary venous obstruction from fibrosing mediastinitis. Int J Cardiol 2006; 108: 418-421.

13 Shapiro BP, Sprung J, Scott K, et al. Cardiovascular collapse induced by position-dependent pulmonary vein occlusion in a patient with fibrosing mediastinitis. Anesthesiology 2005; 103: 661-663.

14 Chazova I, Robbins I, Loyd J, et al. Venous and arterial changes in pulmonary veno-occlusive disease, mitral stenosis and fibrosing mediastinitis. Eur Respir J 2000; 15: 116-122.

15 Nunes $\mathrm{H}$, Humbert M, Capron F, et al. Pulmonary hypertension associated with sarcoidosis: mechanisms, haemodynamics and prognosis. Thorax 2006; 61: 68-74. 